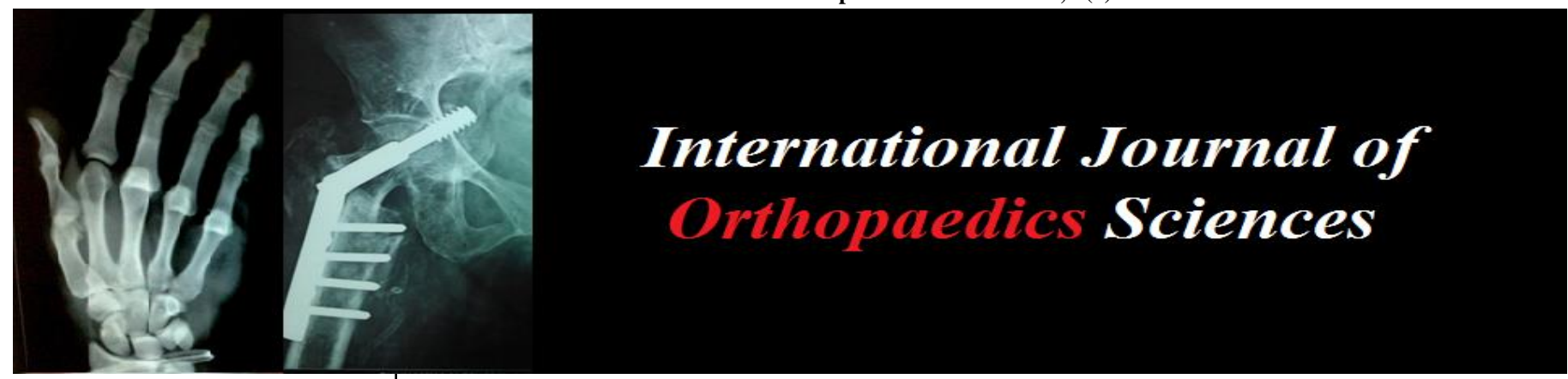

ISSN: $2395-1958$

IJOS 2019; 5(2): 334-338

(C) 2019 IJOS

www.orthopaper.com

Received: 06-02-2019

Accepted: 10-03-2019

Dr. Pradeep D Jadhav

Registrar, Department of Orthopaedics, Manipal Hospital,

Bangalore, Karnataka, India

Dr. Hemant K Kalyan Consultant and HOD,

Department of Orthopaedics, Manipal Hospital, Bangalore,

Karnataka, India

Dr. Sushal Shanthakumar Consultant, Department of Orthopaedics, Manipal Hospital, Bangalore, Karnataka, India

Dr. Samarth Arya Clinical Fellow, Department of Orthopaedics, Wrightington Hospital, United Kingdom

Dr. Harshavardhana V Junior Resident, Department of Orthopaedics, Manipal Hospital, Bangalore, Karnataka, India

Dr. Ajay Kumar SP

Registrar, Department of Orthopaedics, Manipal Hospital, Bangalore, Karnataka, India
Correspondence

Dr. Samarth Arya

Clinical Fellow, Department of

Orthopaedics, Wrightington

Hospital, United Kingdom

\section{Functional outcome following arthroscopic rotator cuff repair with single row technique using bio absorbable anchors: Prospective study}

\author{
Dr. Pradeep D Jadhav, Dr. Hemant K Kalyan, Dr. Sushal Shanthakumar, \\ Dr. Samarth Arya, Dr. Harshavardhana V and Dr. Ajay Kumar SP
}

DOI: https://doi.org/10.22271/ortho.2019.v5.i2g.43

\section{Abstract}

Background: Rotator cuff muscles play an important role in movements of the shoulder joint as well as in stabilizing the joint. The causes of rotator cuff tears are multifactorial. It can be due to degeneration or secondary to trauma. Rotator cuff repairs can be performed through either arthroscopic or open approaches. Complete arthroscopic Rotator Cuff repair have been promising and evolving.

Methodology: Fifty patients between age of 25 to 80 who underwent arthroscopic repair of rotator cuff tears with single row technique using bioabsorbable anchors were analysed for functional outcome and the influence of age, gender and hand dominance as factors influencing functional outcome. The functional outcome of arthroscopic repair of rotator cuff tear with single row technique by using SPADI score and Constant shoulder score.

Results: All the pre-op patients had poor score. After 3 months, $46(92 \%)$ patients were having poor score and $4(8 \%)$ were having fair score. After 6 months, $28(56 \%)$ patients were having fair score, 11 (22\%) patients with good and $11(22 \%)$ patients with poor score. In 1 year follow up, $23(46 \%)$ patients had good score, $22(44 \%)$ patients were having excellent score, $4(8 \%)$ patients with poor and $1(2 \%)$ patients had fair score.

Conclusion: Arthroscopic rotator cuff repair with single row technique demonstrates significant reduction in pain and improvement in shoulder function during medium term follow up. We found that age, gender and association of hand dominance are not significant predictors of outcome of surgery.

Keywords: shoulder, rotator cuff, arthroscopic rotator cuff repair, single row technique

\section{Introduction}

The tendons of Supraspinatus, Infraspinatus, Teres minor and Subscapularis coalesce to form the rotator cuff. The muscles are inseparable, except for subscapularis which is separate and joined to the rest of the cuff via the rotator interval. Rotator cuff muscles together play an important role in movements of the shoulder joint as well as in stabilizing the joint. They lie close to the centre of rotation and their lever arm is shorter. Due to this anatomy it provides stability to the dynamic fulcrum during glenohumeral abduction. A Rotator cuff tear is common cause of pain and disability among adults. A torn Rotator cuff will weaken shoulder. This means that, many daily activities may become painful and difficult to do in several patients but may be largely asymptomatic in some. The causes of rotator cuff tears are multifactorial. However, it is believed that degeneration is an essential factor. However, acute tears can also occur secondary to trauma. In general, the treatment for rotator cuff tears should be clinical, even in cases in which anatomic changes are also present. However, in cases of rotator cuff failures, surgical treatment is indicated. Rotator cuff repairs can be performed through either arthroscopic or open approaches. Although the best procedure for repairing a full thickness Rotator Cuff tear is still controversial, results with most of the studies of complete arthroscopic Rotator Cuff repair have been promising and evolving as a future alternative to traditional open and mini open techniques.

\section{Methodology}

This was a prospective observational study conducted in Manipal Hospital, Bangalore between 
March 2016 to May 2018, to assess the functional outcome of arthroscopic repair of rotator cuff tears with single row technique using bioabsorbable anchors and to assess the influence of age, gender and hand dominance as factors influencing functional outcome using this technique. Fifty patients between age of 25 to 80 years admitted with Rotator cuff tear. The sample size formulae used are as follows:

$$
n_{1}=\frac{\left(\sigma_{1}^{2}+\sigma_{2}^{2} / \kappa\right)\left(z_{1-\alpha / 2}+z_{1-\beta}\right)^{2}}{\Delta^{2}}
$$

The notation for the formulae is:

$$
\begin{aligned}
& n_{1}=\text { sample size of Group } 1 \\
& n_{2}=\text { sample size of Group } 2 \\
& \sigma_{1}=\text { standard deviation of Group } 1 \\
& \sigma_{2}=\text { standard deviation of Group } 2 \\
& \Delta=\text { difference in group means } \\
& K=\text { ratio }=\mathrm{n}_{2} / \mathrm{n}_{1} \\
& Z_{l-a / 2}=\text { two-sided } Z \text { value (eg. } \mathrm{Z}=1.96 \text { for } 95 \% \text { confidence interval). } \\
& Z_{1-\beta}=\text { power }
\end{aligned}
$$

$\mathrm{SD}$ of first group $=0.12$

$\mathrm{SD}$ of second group $=0.15$

$\Delta=$ Difference in mean value $=0.82-0.74=0.08$

$\mathrm{K}=1$
$\mathrm{N}=\underline{(3.99 * 3.99+4.00 * 4.00)(1.96+0.84)^{2}}$

$=45.20=45$

$=45$ samples

By assuming $10 \%$ non-response rate i.e. 4.5. So, sample size was rounded up to 50. Patients with partial tears on MRI, revision rotator cuff repair, greater tuberosity avulsion fracture, current or prior infection, irreparable tears were excluded from the study. All the surgeries were performed by a single orthopaedic shoulder surgeon.

Post operatively, all patients were immobilized with a shoulder pouch and allowed out of the sling for dressing, bathing and post op exercises for 3 weeks. The gentle pendulum exercise was started from the second postoperative day. Patients were discharged from the hospital on the first or second postoperative day. The first outpatient follow-up occurred one week after surgery. Patients were advised pendulum exercises for 3 weeks post operatively, with progressively increasing range. They were then put on active assisted exercises with a stick for the next 3 weeks (4-6 weeks). Active motion was permitted after 6 weeks and physiotherapy advised to improve range of motion and cuff strengthening. Subsequent follow-up visits were at 1, 2, 3, 6, 12 months after surgery. The functional outcome of arthroscopic repair of rotator cuff tear with single row technique by using SPADI score and Constant shoulder score.

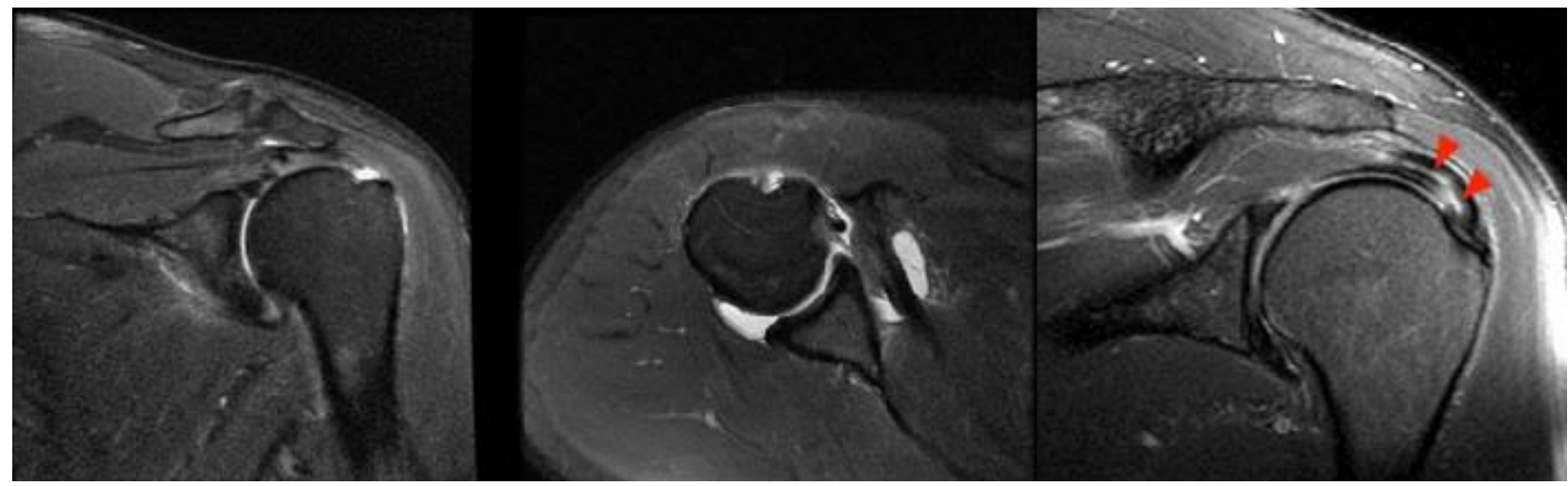

Fig 1: MRI images showing supraspinatus tear in coronal section, subscapularis tear in axial sectio and infraspinatus tear in coronal section

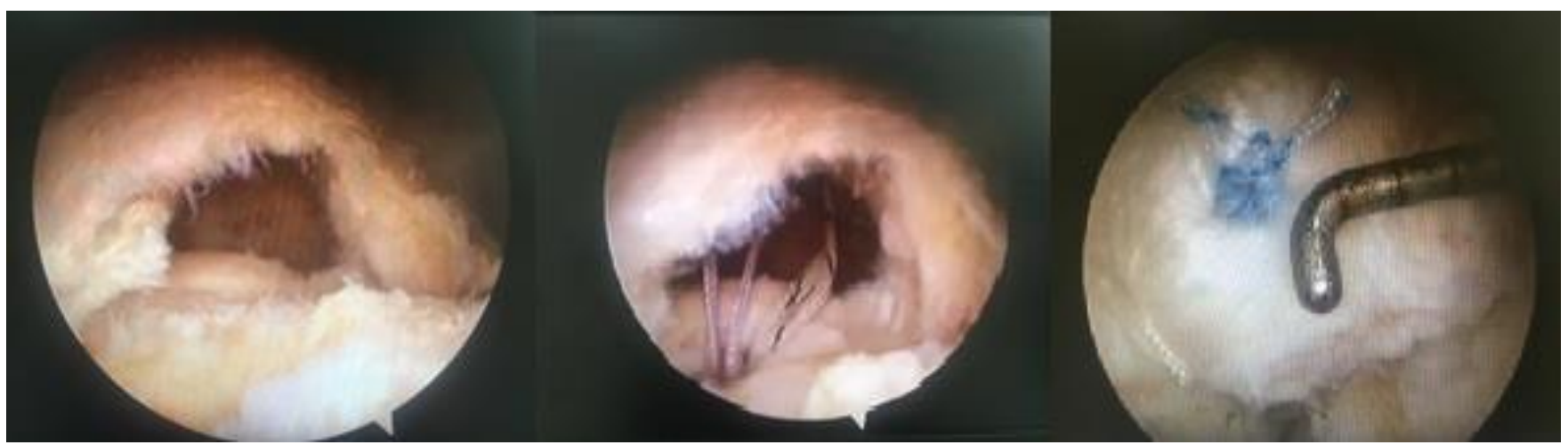

Fig 2: Arthroscopic image of Rotator cuff repair

\section{Results}

Statistical analysis will be done by using descriptive and inferential statistics using Student's paired and unpaired t test, and software used in the analysis were SPSS 22.0 version and Graph Pad Prism 6.0 version and $\mathrm{p}<0.05$ is considered as level of significance.

In our study total 50 patients were included and were divided into five groups according to their age groups. Twenty-nine patients were males and 21 patients were females of which, $90 \%(45 / 50)$ were right handed and $10 \%(5 / 50)$ patients were left handed. Out of 45 right handed patients, 41 patients got operated on right side itself and 4 patients got operated on non-dominant side i.e. left side. Out of 5 left handed patients, 4 patients got operated on left side itself and 1 patient got 
operated on non-dominant side i.e. right side.

Table 1: Age wise distribution of patients

\begin{tabular}{|c|c|c|}
\hline Age Group (years) & No of patients & Percentage \\
\hline $25-34$ yrs & 2 & 4 \\
\hline $35-44$ yrs & 3 & 6 \\
\hline $45-54$ yrs & 11 & 22 \\
\hline $55-64$ yrs & 20 & 40 \\
\hline $65-74$ yrs & 14 & 28 \\
\hline Total & 50 & 100 \\
\hline Mean \pm SD & $57.52 \pm 10.67(24-72$ yrs $)$ \\
\hline
\end{tabular}

MRI reports showed that all patients (100\%) had complete tear of supraspinatus muscle. Out of 50 patients, $39(78 \%)$ patients had only supraspinatus tear, 6 (12\%) had supraspinatus with infraspinatus muscle tear and $5(10 \%)$ patients had supraspinatus with subscapularis muscle tear. Eleven patients $(22 \%)$ had small size $(<1 \mathrm{~cm}), 29(58 \%)$ patients had medium size $(1 \mathrm{~cm}-3 \mathrm{~cm})$ and $10(20 \%)$ patients had large tear $(>3 \mathrm{~cm})$. Average length of tear was $18.28 \pm 10.12$.

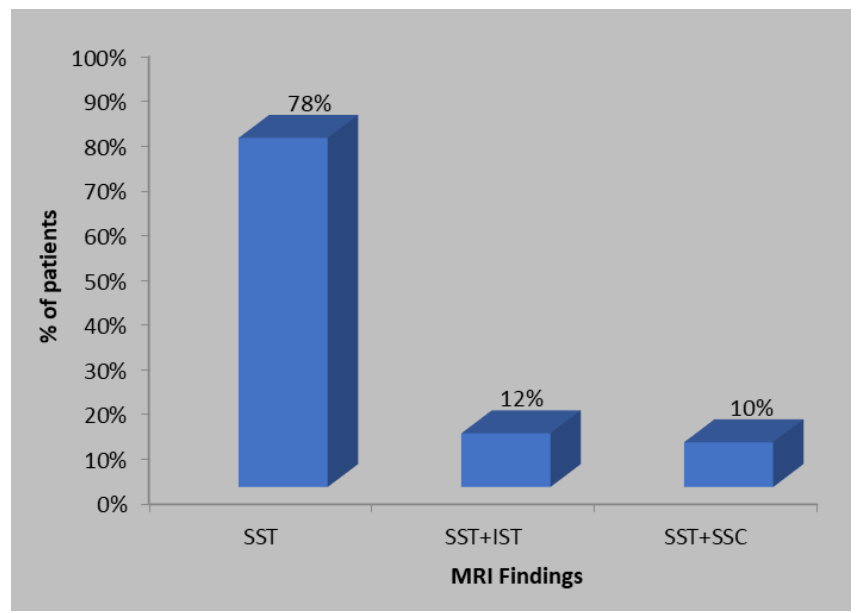

Graph 1: Distribution of patients according to MRI findings

In the MRI, we observed that, 46 patients had same findings with MRI and arthroscopy. Thirty-nine (78\%) patients had only supraspinatus tear, 6 patients $(12 \%)$ had supraspinatus with infraspinatus muscle tear and $5(10 \%)$ patients had supraspinatus with subscapularis muscle tear. Whereas, during arthroscopic we observed that, 35 (70\%) patients had only supraspinatus tear, $9(18 \%)$ had supraspinatus with infraspinatus muscle tear and $6(12 \%)$ had supraspinatus with subscapularis muscle tear.

Table 2: Correlation between MRI and arthroscopic findings

\begin{tabular}{|c|c|c|c|}
\hline Findings & MRI Findings & $\begin{array}{c}\text { Arthroscopic } \\
\text { Findings }\end{array}$ & \multirow{2}{*}{$\chi$ 2-value } \\
\hline SST & $39(78 \%)$ & $35(70 \%)$ & \multirow{2}{*}{0.90} \\
\cline { 1 - 3 } SST+IST & $6(12 \%)$ & $9(18 \%)$ & 0.90 \\
\cline { 1 - 2 } SST+SSC & $5(10 \%)$ & $6(12 \%)$ & p 0.63, NS, $p>0.05$ \\
\hline Total & $50(100 \%)$ & $50(100 \%)$ & \\
\hline
\end{tabular}

Pre-op mean pains core was $88.64 \pm 3.43$ and post-op which was $46.88 \pm 5.7$ in 3 month follow up, 10.36 2.6 in 6 month follow up, $3.16 \pm 1.56$ in 1 year follow up. Pre-op mean disability score was $82.17 \pm 4.27$ and post-op which was $44.09 \pm 3.55$ in 3 month follow up, 23.92 3.67 in 6 month follow up, $5.62 \pm 2.49$ in 1 year follow up.
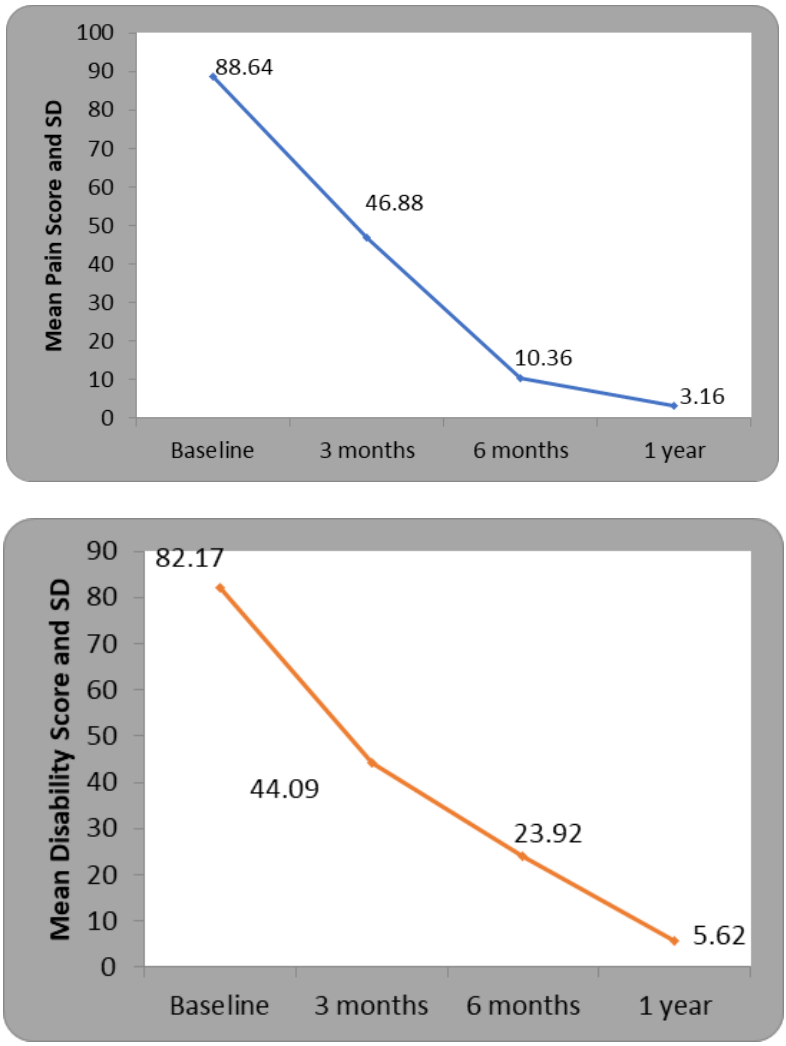

Graph 2: Comparison of pain and disability score at pre-op, 3 months, 6 months and 1 year

Pre-op mean SPADI score was 84.71 (S.D. \pm 3.47 ) and postop which was 44.68 (S.D. \pm 3.61) in 3 month follow up, 18.78 (S.D. \pm 2.32) in 6 month follow up, 4.97 (S.D. \pm 2.54 ) in 1 year follow up. Pre-op mean constant shoulder score was $32.68 \pm 3.87$ and post-op which was $56.78 \pm 8.38$ in 3 month follow up, $66.22 \pm 7.17$ in 6 month follow up, $78.72 \pm 6.88$ in 1 year follow up.
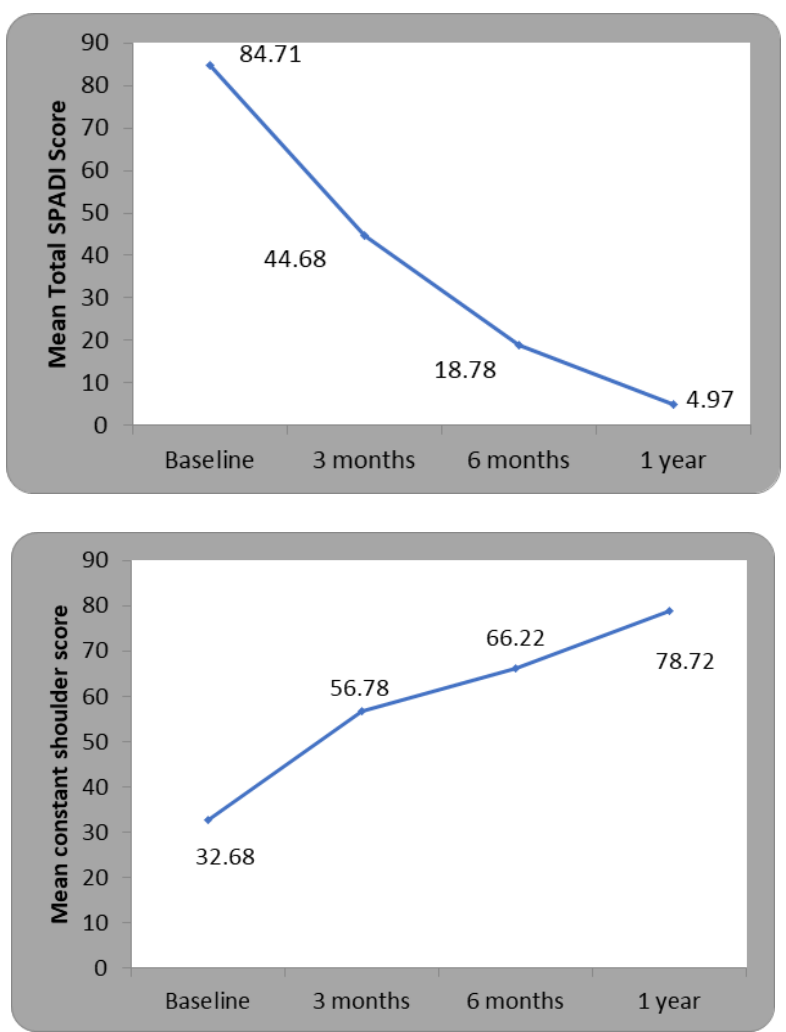

Graph 3: Comparison of total SPADI score and constant shoulder score at pre-op, 3 months, 6 months and 1 year 
All the pre-op patients had poor score. After 3 months, 46 (92\%) patients were having poor score and $4(8 \%)$ were having fair score. After 6 months, 28 (56\%) patients were having fair score, $11(22 \%)$ patients with good and $11(22 \%)$ patients with poor score. In 1 year follow up, $23(46 \%)$ patients had good score, 22 (44\%) patients were having excellent score, $4(8 \%)$ patients with poor and $1(2 \%)$ patients had fair score.

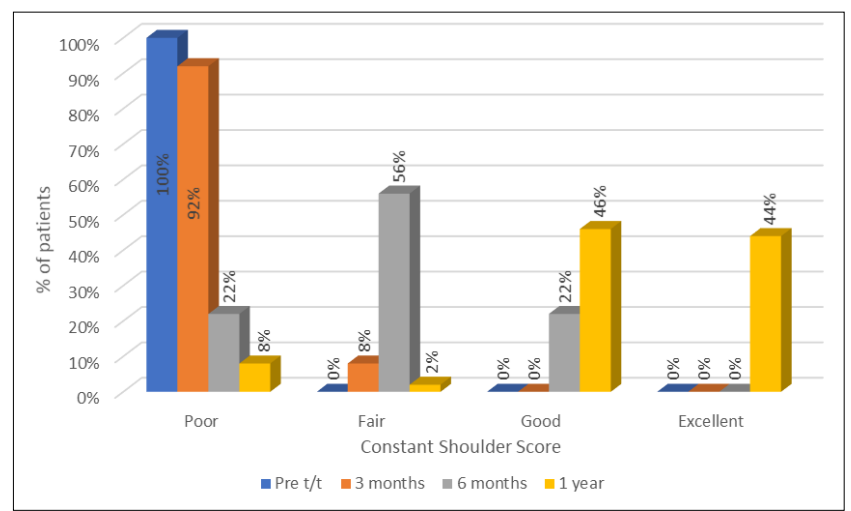

Graph 4: Comparison of constant shoulder score grading at pre t/t, at 3 months, 6 months and 1 year

Pain score for patient with right hand dominance was $88.53 \pm$ 3.57 preoperatively and final Pain score $3.24 \pm 1.61$ at the end of one year. And average Pain score for patient with left hand dominance was $89.6 \pm 1.61$ preoperatively and $2.4 \pm 0.89$ at the end of one year.

Disability score for patient with right hand dominance was $82.13 \pm 4.14$ preoperatively and final Disability score $5.61 \pm$ 2.50 at the end of one year and average Disability score for patient with left hand dominance was 82.50 \pm 2.50 preoperatively and $5.74 \pm 2.59$ at the end of one year.

Preoperatively average SPADI score for patients with small, medium and large tear was 83.98, 84.29 and 86.7 respectively. And postoperatively at the end of 1-year, average SPADI score for patients with small, medium and large tear was 4.26, 5.24 and 4.99 respectively. Preoperatively average constant shoulder score for patients with small, medium and large tear was 33.72, 33.2 and 30 respectively and postoperatively at the end of 1-year, average constant shoulder score for patients with small, medium and large tear was $82,80.51$ and 69.9 respectively. Three patients had complications, of which one patient had a re-tear and two patients had superficial infection.

\section{Discussion}

In our study total 50 patients were included and were divided into five groups according to their age groups. Majority of the patients were in age group of 55-64 years which suggests that, a greater number of patients belong to higher age groups and it correlates with the fact that rotator cuff tear is due to the tendon degeneration occurring due to aging ${ }^{[1]}$. Lehman $\mathrm{C}$ et $a l^{(2)}$ concluded that the incidence of rotator cuff tears had a linear correlation with increasing age and that the prevalence was 6 percent in individuals less than 60years of age and the prevalence increased to 30 percent in individuals more than 60 years of age. Overall prevalence was found to be $17 \%$. We had 29 males and 21 females. All of them were having symptomatic rotator cuff tears. It is seen that there is no significant difference within the population according to gender which suggests that the occurrence of rotator cuff tears is not gender dependant. E. Lawrence Keyes in his study mentioned that gender did not influence the occurrence and distribution of rotator cuff tears / lesions ${ }^{[3]}$. C Milgrom and his colleagues ${ }^{[4]}$, concluded in their study on 90 individuals that there was no statistically significant gender difference which affected the prevalence of asymptomatic rotator cuff tears. In our study, total 45/50 (90\%) were right handed and $5 / 50(10 \%)$ patients were left handed. It is seen that there is significant difference within the population according to hand dominance which suggests that the occurrence of rotator cuff tears is hand dominance dependant. Yamamoto A et al. ${ }^{\text {[5] }}$ concluded in their study that dominant arm was a significant risk factor for occurrence of rotator cuff tear.

MRI finding of $92 \%$ patients were matching with arthroscopic finding. In three patients, arthroscopy showed infraspinatus muscle involvement which was not showed in MRI report and in one patient through arthroscopic we observed that extra subscapularis tear which was not showed in MRI report. This subscapularis tear was small, so didn't require repair. In some patient arthroscopy also showed fraying and degeneration of long head of biceps. There was no statistically significant difference between MRI finding and arthroscopic finding ( $p$ > $0.05)$. Currently, arthroscopy is considered the "reference standard" for the diagnosis of shoulder pathologies. However, arthroscopy is an invasive procedure that requires hospitalisation and anaesthesia and carries a small risk of complications such as infection, damage to adjacent structures such as brachial plexus, as well as anaesthesia related complications ${ }^{[6]}$. MRI with its excellent soft tissue resolution and multiplanar imaging capabilities has now emerged as the investigation of choice in the preoperative evaluation of rotator cuff diseases ${ }^{[7]}$.

The SPADI score was 84.71(S.D. \pm 3.47 ) and post-op which was 44.68 (S.D. \pm 3.61 ) in 3 month follow up, 18.78 (S.D. \pm 2.32 ) in 6 month follow up, 4.97 (S.D. \pm 2.54 ) in 1 year follow up. In this study, there is statistically significant reduction in pain and disability i.e. SPADI Score by the end of 1 year follow up ( $\mathrm{P}$ value $=0.0001)$. Pre-op mean Constant Shoulder Score was 32.68 (S.D. \pm 3.87 ) and post-op which was 56.78 (S.D. \pm 8.38 ) in 3 month follow up, 66.22 (S.D. \pm 7.17 ) in 6 month follow up, 78.72 (S.D. \pm 6.88 ) in 1 year follow up. In this study, there is statistically significant reduction in pain and disability i.e. SPADI Score by the end of 1 year follow up ( $\mathrm{P}$ value $=0.0001)$. The relation between age, gender and association of hand dominance with SPADI and constant shoulder score was not statistically significant $(p>0.05)$. In our study, preoperatively average SPADI score for patients with small, medium and large tear was $83.98,84.29$ and 86.7 respectively and postoperatively at the end of 1-year, average SPADI score for patients with small, medium and large tear was 4.26, 5.24 and 4.99 respectively. We found there no statistically significant relation between size of tear and SPADI score $(\mathrm{p}>0.05)$. In our study, preoperatively average constant shoulder score for patients with small, medium and large tear was 33.72, 33.2 and 30 respectively and postoperatively at the end of 1-year, average constant shoulder score for patients with small, medium and large tear was $82,80.51$ and 69.9 respectively. We found that patients with small and medium tear patients reported similar outcomes, however those with large tears showed less favourable outcome based on Constant shoulder score.

Complications following arthroscopic rotator cuff repair included shoulder stiffness, failure of healing (re-tear), infection, reflex sympathetic dystrophy, deep venous thrombosis, and death. In our study, one patient sustained retear and two patients got superficial infection. The patient 
with re-tear revision surgery. Patients with superficial infection was treated with antibiotics and regular dressing.

\section{Conclusions}

Our study shows that arthroscopic rotator cuff repair with single row technique demonstrates significant reduction in pain and improvement in shoulder function during medium term follow up. We found that age, gender and association of hand dominance are not significant predictors of outcome of surgery. There is a strong association between the occurrence of rotator cuff tears and increasing age and hand dominance. There is no apparent gender bias in the occurrence of rotator cuff tears. MRI findings showed that most of the patients had supraspinatus tear alone involving central tendon with medium size tear. Patients with small and medium tear patients reported similar outcomes, however those with large tears showed less favourable outcome based on Constant shoulder score.

Although our results related to structural integrity support arthroscopic single-row technique using suture anchor fixation, we did not perform a randomized direct comparison of single-row, arthroscopic double-row, and open double-row repair, hence we are unable to definitively conclude that this technique for rotator cuff repair is superior to all others. Evaluation of healing of cuff can be documented through regular postoperative MRI or USG scans will help in determining the healing rates of rotator cuff following the arthroscopic repair. A bigger sample size will eliminate the limitations and would help to extrapolate the result on to the general population.

\section{References}

1. Brand RA. Biographical Sketch: John Gregory Smith, FRCS. Clin Orthop Relat Res. 2010; 1;468(6):1469-70.

2. Lehman C, Cuomo F, Kummer FJ, Zuckerman JD. The incidence of full thickness rotator cuff tears in a large cadaveric population. Bulletin (Hospital for Joint Diseases (New York, NY). 1994; 54(1):30-1.

3. Wilson CL, Duff GL. Pathologic study of degeneration and rupture of the supraspinatus tendon. Arch Surg. $1943 ; 47(2): 121-35$.

4. Milgrom C, Schaffler M, Gilbert S, Van Holsbeeck M. Rotator-cuff changes in asymptomatic adults. The effect of age, hand dominance and gender. J Bone Joint Surg Br. 1995; 77(2):296-8.

5. Atsushi Yamamoto MD, Kenji Takagishi MD, PhD, Toshihisa Osawa MD, PhD Takashi Yanagawa MD, $\mathrm{PhD}$, Daisuke Nakajima, PhD Prevalence and risk factors of a rotator cuff tear in the general population J Shoulder Elbow Surg. 2010; 19:116-120.

6. Bhatnagar A, Bhonsle S, Mehta S. Correlation between MRI and arthroscopy in diagnosis of shoulder pathology. J Clin Diagn Res. 2016; 10(2):RC18-RC21.

7. Opsha O, Malik A, Baltazar R, Primakov D, Beltran S, Miller TT et al. MRI of the rotator cuff and internal derangement. Eur J Radiol. 2008; 68(1):36-56. 